MATTERS ARISING OPEN

\title{
Machine learning detection of obstructive hypertrophic cardiomyopathy using a wearable biosensor
}

\author{
R. Ueno ${ }^{1 *}$, H. Matsui ${ }^{2}$ and L. $\mathrm{Xu}^{2}$ \\ ARISING FROM Green et al. npj Digital Medicine https://doi.org/10.1038/s41746-019-0130-0 (2019)
}

npj Digital Medicine (2019)2:120; https://doi.org/10.1038/s41746-019-0186-x

Green et al. (June 24 issue) $^{1}$ developed a machine learning classifier of hypertrophic cardiomyopathy (HCM) patients using a noninvasive optical sensor incorporated in commercial smart watches. The study included 83 patients (19 patients with HCM and 64 healthy controls) and a machine learning classifier was trained with Leave-One-Group-Out cross-validation with nested hyperparameter tuning, achieving a C-statistic of $0.99(95 \% \mathrm{Cl}$ : 0.99-1.00) for 82 patients.

While these results are promising and persuasive enough to facilitate further trial to assess its performance in a larger cohort, we would argue a few statistical concerns with the study. First, the authors argued that they have performed 68-fold, instead of 82fold cross-validation for their Leave-One-Group-Out crossvalidation. Further clarification might be required to avoid any confusion for readers. Second, the confusion matrix includes all the 82 patients from both train and test data, while this table should ideally include only test data. ${ }^{2}$ Inclusion of train group in this matrix might result in the over-estimate performance of the model.

We believe those findings might not invalidate the insightful work by Green et al. Further clarification of such concerns might further reinforce this study to function as a foundation for a larger study.

Received: 25 June 2019; Accepted: 1 October 2019;

Published online: 10 December 2019

\section{REFERENCES}

1. Green, E. M. et al. Machine learning detection of obstructive hypertrophic cardiomyopathy using a wearable biosensor. Npj Digit. Med. 2, 57 (2019).

2. Chicco, D. Ten quick tips for machine learning in computational biology. BioData Min. 10, 35 (2017).

\section{AUTHOR CONTRIBUTIONS}

All authors provided important contributions to the conception of and the drafting of this correspondence. All authors reviewed the content, revised the manuscript, and approved the final version. R.U. led the drafting of this correspondence.

\section{COMPETING INTERESTS}

The authors declare no competing interests.

\section{ADDITIONAL INFORMATION}

Correspondence and requests for materials should be addressed to R.U.

Reprints and permission information is available at http://www.nature.com/ reprints

Publisher's note Springer Nature remains neutral with regard to jurisdictional claims in published maps and institutional affiliations.

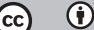

Open Access This article is licensed under a Creative Commons Attribution 4.0 International License, which permits use, sharing, adaptation, distribution and reproduction in any medium or format, as long as you give appropriate credit to the original author(s) and the source, provide a link to the Creative Commons license, and indicate if changes were made. The images or other third party material in this article are included in the article's Creative Commons license, unless indicated otherwise in a credit line to the material. If material is not included in the article's Creative Commons license and your intended use is not permitted by statutory regulation or exceeds the permitted use, you will need to obtain permission directly from the copyright holder. To view a copy of this license, visit http://creativecommons. org/licenses/by/4.0/.

(c) The Author(s) 2019

\footnotetext{
${ }^{1}$ Frankston Hospital, Melbourne, Australia. ${ }^{2}$ The University of Tokyo, Tokyo, Japan. *email: ryo.ueno@monash.edu
} 